ISSN 1112-9867

\title{
A NOVEL PERCEPTUALLY ADAPTIVE IMAGE WATERMARKING SCHEME BY SELECTING ADAPTIVE THRESHOLD IN DHT DOMAIN
}

\author{
E. Moeinaddini
}

Department of Engineering, University of Jiroft, Jiroft, Iran

Published online: 15 May 2016

\begin{abstract}
This paper proposed a novel image watermarking technique by applying the characteristics of the human visual system, in Hadamard transform domain. Statistical information measures were used to select proper blocks for data embedding. Watermark was embedded by the modification of Discrete Hadamard transform (DHT) coefficients of selected blocks. Threshold and modification value were selected adaptively for each image block, which improved robustness and transparency. The proposed algorithm was able to withstand a variety of attacks and image processing operations like rotation, cropping, noise addition, resizing, lossy compression and etc. The experimental results showed good performance of the proposed scheme in comparison with some of the recently reported watermarking techniques.
\end{abstract}

Key words: Digital image watermarking, Hadamard transform, Entropy, Lossy compression, Adaptive Threshold.

\footnotetext{
Author Correspondence, e-mail: author@yahoo.com

doi: http://dx.doi.org/10.4314/jfas.v8i3s.269
}

\section{INTRODUCTION}

Digital watermarking is a process of hiding metadata containing information about the digital content in any multimedia such that the inserted data becomes part of the media. This technique is used for different purposes such as establishment and proving of ownership rights, prevention of illegal replication, facilitating content authentication etc[1]. The earliest watermarking 
techniques mainly focused on the spatial domain, such as least significant bit (LSB) and vector quantization (VQ)[2].These methods were not robust enough against usual image processing attacks and had little capacity[3].Frequency domain is another approach. These techniques mostly used different transforms such as discrete Fourier transform (DFT) [4],Fourier-Mellin[5, 6], spread spectrum (SS) [7],contourlet transform [8, 9]discrete cosine transform (DCT)[10, 11] and wavelet transformations $[12,13]$.

Among these transforms, DCT and DWT were mostly used for the implementation of compression resilient watermarking schemes, since the most common image compression techniques based on DCT and DWT, were JPEG and JPEG 2000. [14, 15].

Ramkumar.et.al.[16]demonstrated that Hadamard transforms, provides higher resiliency at high degree (low quality) JPEG and JPEG 2000 compression compared to DCT and DWT because of the small values of the standard deviation for the noise processing[17].

Here we present a brief review related to recently published DHT, DCT and DWT based and some HVS based watermarking techniques. Das et al.[18] proposed a watermarking scheme in DCT domain. They embedded watermark's bits by changing DCT coefficients from two neighboring blocks and brought the difference from the adjacent block coefficient in a specified range. A perceptual model based DCT domain QIM watermarking was proposed by Li and Cox [19] to improve imperceptibility and robustness against volumetric scaling. S.P. Maity et al.[17] proposed a watermarking scheme in DHT domain based on HVS and spread transform (ST). The Spread transform scheme was implemented using the transform coefficients of both the watermark signal and the host image. A wavelet-based watermarking scheme was proposed by Lin and Lin [13] which involved generating the secret key during the embedding process, using local features extracted from the perceptually prominent components of the wavelet transformed host image. Similarly, Kumsawat et al.[20] used multi-wavelet transform and genetic algorithms (GAs) to propose an algorithm for optimization in zero-rate SS watermarking. The major problem with some of these watermarking techniques is that they are not robust enough against different types of attacks. Some of them embed watermark without considering human visual system (HVS), some of these techniques are complicated to implement in real-time. Moreover in most of the existing watermarking algorithms threshold has been adjusted manually and did not obtained in an optimal way. The threshold for watermarking algorithm determines the strength 
factor that controls the robustness and imperceptibility. Therefore, for having an efficient algorithm we have to find the suitable values of this parameter.

In this paper, a novel HVS based watermarking scheme using block wise discrete Hadamard transform (DHT) was proposed. The algorithm focuses on the selection of the blocks to embed watermark information such that data imperceptibility is well preserved. The use of DHT offers better robustness against noise addition, and low visual distortion, when HVS characteristics with entropy masking are used. But the novelity of this scheme is in adaptive selection of threshold, the threshold for each block was selected automatically depending upon the block's informations, to achieve a better performance. Experimental results showed improved results in case of fidelity and robustness against some of common attacks such as median filtering, JPEG compression, cropping, rotation and resizing in comparison with other DHT, DCT and DWT base methods.

The paper is organized as follows: Section 2 involves the explanation on how to find proper blocks for data hiding using Entropy. Hadamard transform is briefly introduced in section 3. Section 4 explains the proposed embedding and extracting methods. Experimental results of the proposed work are given in section 5; followed by conclusion in section 6 .

\section{FINDING PROPER BLOCKS FOR DATA HIDING}

This section explains the average information (entropy), its mathematical forms and its usefulness to select suitable blocks for and robust watermarking. The human visual system (HVS) extracts perceptual information from a group of pixels not from the single pixel alone whenever a picture is looked at. There exists a special correlation between the neighboring pixels in images, known as structural information. Entropy is a measure of unpredictability of information content and is a good measure for spatial correlation of neighboring pixels. Shannon [21] formula utilized this information in calculating entropy. It shows in Eq.(1)

$$
E=-\sum_{i=1}^{n} p_{i} \log _{p^{i}}
$$

where $p_{i}$ is the probability of occurrence of the event $i, 0 \leq p_{i} \leq 1$ and $\sum_{i=1}^{n} p_{i}=1$ [21].

His entropy considered the signal as a along sequence of symbols. This entropy depended on the relative occurrence of the symbols, irrespective of their position of occurrence. Pal and Pal in 
[22] defined average edge information as an exponential form of entropy formula that can capture 2D spatial correlation of images better than Shannon's entropy. entropy defined in [22] as

$$
E=\sum_{i=1}^{n} p_{i} \exp ^{u i}=\sum_{i=1}^{n} p_{i}{\operatorname{exp^{1}}}^{1-p i}
$$

Where $u_{i}=\left(1-\mathrm{p}_{\mathrm{i}}\right)$ is uncertainty of the pixel value.

In the present work, the average gray information Eq. (1) and average edge information Eq. (2) were used to find robust and resilience blocks of images for data hiding. These two quantities for each block were computed and added. Following this, was the sorting of the values in ascending order, as a linear chain. This chain was divided into 3 parts: low, medium and high-informative blocks. The medium-informative blocks were selected for embedding process to satisfy both imperceptibility and robustness requirements[17].

\section{OVERVIEW OF 2D-HADAMARD TRANSFORMATION}

This section highlights a brief review of Hadamard Transformation on images and its advantages for watermarking technique. 2D-Hadamard Transform is non-sinusoidal orthogonal transformation which can reduce the band width of signals, thereby reducing the storage requirement. However, it is easy to calculate because of the use of real addition and subtraction, hence its suitability for implementing real-time watermarking systems.

This Transformation is based on Hadamard Transform matrix. The Hadamard matrix is a $2^{\mathrm{n}} \times 2^{\mathrm{n}}$ array of -1 and +1 with the rows and columns orthogonal to one another. The elements of the transform matrix are simple: they are binary, real numbers. Therefore, the forward and inverse Hadamard transforms can be calculated using simple rules. Because of orthogonality of its rows and columns, $\mathrm{H}_{\mathrm{n}}$ matrix has some special features. One of its significant features is shown in (3):

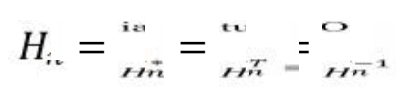

Where $\mathrm{H}_{\mathrm{n}}$ is Hadamard Matrix, $H_{n}^{T}$ is Transpose of Hadamard matrix, $H_{n}^{-1}$ is Inverse Hadamard matrix and $H_{n}^{*}$ is Conjugate Hadamard matrix. 
For $\mathrm{N}=2$, the Hadamard matrix, $H_{1}$, is called a core matrix, which is defined as

$$
\text { is su a } 1 \text { = matr }
$$

The Hadamard matrix of the order $\mathrm{n}$ is generated in terms of Hadamard matrix of order $\mathrm{n}-1$ using Kronecker product, $\otimes$, as

$$
{ }_{m i n}={ }_{m-1} \otimes_{n i 1}
$$

Or

$$
H^{n}=\left[\begin{array}{ll}
H^{n-1} & -H_{-H}^{H-1} \\
H n-1 & -H n-1
\end{array}\right]
$$

Finally the 2D-Hadamard transform of an image is given by

$$
V=\frac{H n}{N} \frac{U H_{n}}{N}
$$

where $\mathrm{U}$ represents the original image and $\mathrm{V}$ the transformed image.

Since $H_{n}$ has $\mathrm{N}$ orthogonal rows $H_{n} \times H_{n}=\mathrm{N} \times \mathrm{I}$ (I is the identity matrix) and $H_{n} \times H_{n}=\mathrm{N} \times H_{n} \times H_{n}^{-1}$, thus $H_{n}^{-1}=H_{n} / N$

Hence the inverse Hadamard transform can be calculated using (8):

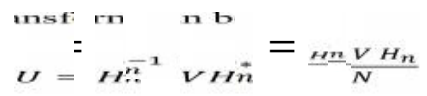

In the proposed algorithm, the transformation process is carried out on $4 \times 4$ blocks using the third order Hadamard transform matrix [23].

\section{PROPOSED ALGORITHM}


For embedding watermark in an $\mathrm{M} \times \mathrm{N}$ image DHT coefficients of medium-informative blocks and entropy concepts are utilized. Every DHT coefficient of the medium-informative blocks shown by $B_{i}(x)(1 \leq x \leq 16), x$ is index for each DHT coefficient in a block and $(1 \leq i \leq \mathrm{M} \times$ $\mathrm{N} /(16 \times 3)) i$ is an index of medium-informative blocks. For embedding each watermark bit, the relationship of two coefficients in a block was modified. As we used medium-informative blocks of size $4 \times 4$ total number of useful blocks for an $M \times N$ image is $M \times N /(16 \times 3)$ so if our test image was $512 \times 512$ the watermark could be a string of maximum 5461 bits or an $73 \times 73$ binary image. In the fallowing sub sections we explained the embedding and extracting process of proposed algorithm.

\subsection{Watermark Embedding}

The watermark embedding process is performed in the following steps:

step1: image was divided into $4 \times 4$ non-overlapping blocks. An image of $\mathrm{M} \times \mathrm{N}$ will have $\mathrm{M} \times \mathrm{N} / 16$ blocks if $\mathrm{M}$ and $\mathrm{N}$ are multiples of 4 .

step2: Medium-informative blocks were found and selected according to section 2. Positions of medium-informative blocks were saved in a secret file for extracting process.

step3: The medium-informative blocks were scanned sequentially and each block is transformed using discrete Hadamard transform.

step4: The watermark message or image is selected and changed to bits string. Bits were permuted and the permutation key saved in a secret file.

step5: Each watermark bits is embedded in a medium-informative block by changing the relation between two DHT coefficients say $B_{i}(a)$ and $B_{i}(b)(5 \leq a, b \leq \leq 16)$ a and $\mathrm{b}$ are two coefficients in blocks. We don't select these coefficients from the first row in order to have less degradation in image. In this work $\mathrm{a}$ and $\mathrm{b}$ were selected as 6 and 11 respectively. We assume that $B_{i}(a)$ is greater than $B_{i}(\mathrm{~b})$ otherwise we exchange these values. For embedding a watermark bit if the binary watermark bit $w_{i}=1$, then the condition $B_{i}(a)-$ $B_{i}(\mathrm{~b}) \geq T h_{i}$ must hold and if the watermark bit $w_{i}=0$, the condition $B_{i}(a)-B_{i}(\mathrm{~b})<$ $T h_{i}$ must hold. If conditions are violated, the $B_{i}(a)$ should be modified based on the following rules(9)-(12) 


$$
\begin{aligned}
& \begin{array}{c}
\left.{ }_{i} w_{i}=1 \quad \& E_{{ }^{i}(}(a)-B^{2}-b\right) \\
<F h_{i},
\end{array} \\
& \text { then } B_{i}^{\prime}(a)=B_{i}(a)+\Delta \\
& +T h_{i} \\
& \text { if } \begin{array}{c}
w_{i}=0 \& B_{i}\left({ }^{-}{ }^{-}\right. \\
\geq T h_{i},
\end{array} \\
& \text { then } B_{i}^{\prime}(a) \\
& =B_{i}(a)-\Delta \\
& -T h_{i} \\
& =_{B t(a)}-{ }_{B t(b)}^{T x_{t}} \\
& { }_{T h}^{-B}{ }^{-}{ }^{-} \\
& =\frac{1}{N c-4} \sum_{x=5}^{N c} B_{i}(x)
\end{aligned}
$$

where $T h_{i}$ is the mean value of all coefficients except the first row in block $i$ and $N c$ is the number of coefficients in block here it is equal to 16. Coefficient's values of first row in blocks are quite higher than others so we didn't conceder those for computing $T h_{i}$.

step6: An inverse Hadamard Transformation applied for blocks.

step7: blocks were integrated to display the watermarked image.

\subsection{Watermark Extraction}

Watermark extraction is a blind process therefore the original image is not necessary for extraction. For extracting watermark, the watermarked image, the medium-informative blocks chain and the key used for permutation of watermark bits were needed. The extraction process is performed in the following steps: 
step1: The watermarked image is divided into $4 \times 4$ non-overlap blocks.

step2: The medium-informative blocks are obtained using information in the secret file.

step3: Fast Hadamard Transformation is applied to the medium-informative blocks.

step4: Compute $T h_{i}$ for each medium-informative blocks using equation (12).

step5: Each bit of watermark w' was extracted using equation (13).

$$
\begin{aligned}
& \text { wi } \\
& =\left\{\begin{array}{ll}
\text { if } B_{i}(a)-B_{i}(\mathrm{~b}) \geq T h_{i} \\
\text { d } & \text { if } B_{i}(a)-B_{i}(\mathrm{~b})<T h_{i}
\end{array} \mid\right. \text { (13) }
\end{aligned}
$$

By repeating the step 5, all watermark bits were extracted.

step6: The inverse permutation is applied on bits using permutation key in secret file to obtain the watermark.

Block diagram of proposed algorithem is depicted in Figure 1.

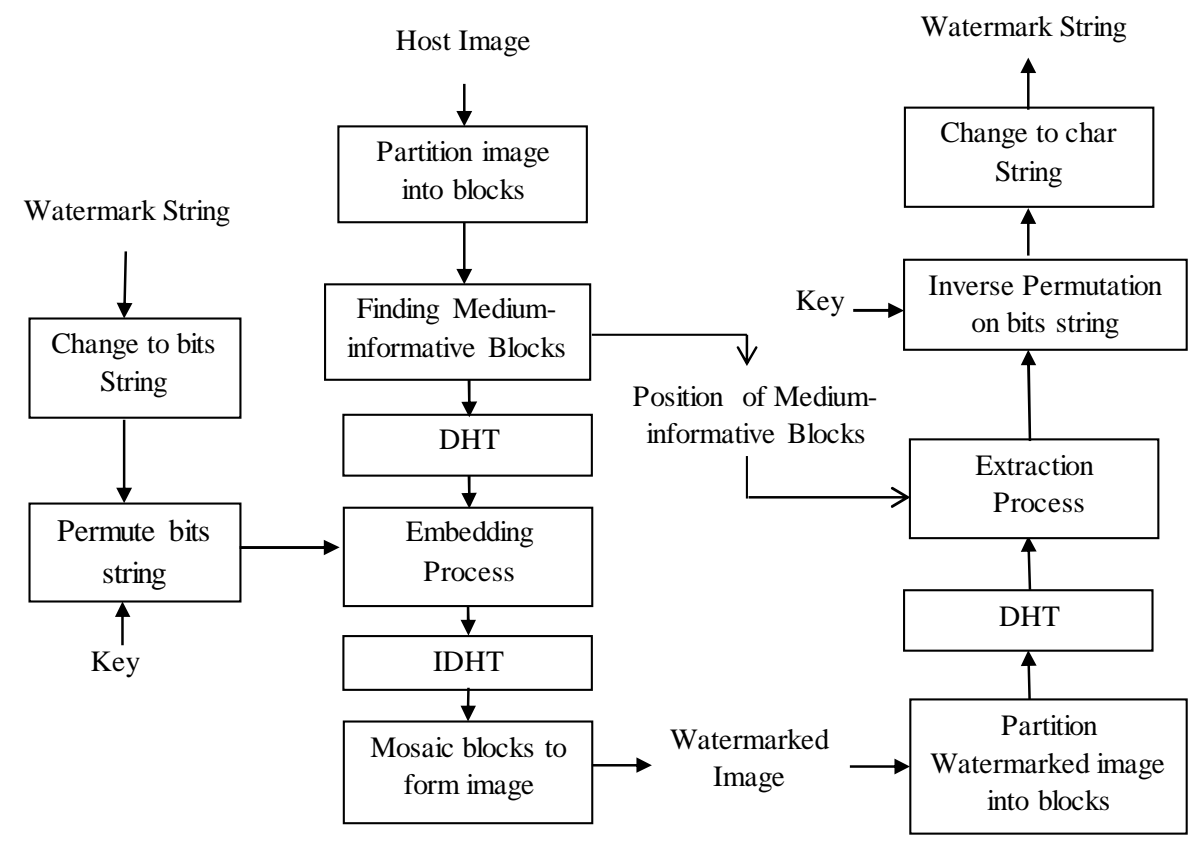

Fig.1. Block diagram representation of the proposed watermarking scheme. 


\section{SIMULATION RESULTS OF PERFORMANCE}

This section describes image fidelity, robustness performance and comparison with other existing watermarking methods. The proposed work was implemented on dual core CPU with 2 GB RAM configured system using MATLAB version R2012a. The most commonly used test images were utilized for the purpose of experimentation. To evaluate performance of the proposed watermarking method, test images of size $512 \times 512$ were used and a watermark message of 4096 bits length embedded. However, this watermark message can be replaced with a $64 \times 64$ binary image.

\subsection{Imperceptibility of Proposed Scheme}

The quality of watermarked images and extracted watermarks were measured through Peak Signal to Noise Ratio (PSNR)[24] as a distortion measure for the watermarked image under inspection, with respect to the original host image.The peak signal to noise ratio is a ratio between the original image and the modified image in decibels. The root mean square error (RMSE) between original image I and modified images $\overline{\mathrm{I}}$ was calculated using Eq.(14) and substituted in Eq.(15) to calculate peak signal to noise ratio:

$$
\begin{aligned}
& \frac{{ }^{L S E}}{\sqrt{\frac{1}{m_{\eta}}-\sum_{n, n}[I(i, j)}-\overline{\bar{I}(i, j)]}} \overline{\overline{2}}= \\
& P S N R=10 \log 10\left(255^{2} / R M S E\right)
\end{aligned}
$$

where $\mathrm{m}$ and $\mathrm{n}$ are width and height of the cover image respectively.

The original test images and their corresponding watermarked images are shown in Figure 2. It can be seen that the watermarked images preserved good visible quality. The obtained results of the comparison of PSNR value between the watermarked image and the original image for different test images with S.P.Maity et al.[17], Ho et al.[23], Lin and Lin[13], and Paquet et al. [25] methods are reported in Table 1. The results showed that the proposed method offers better data imperceptibility compared to other methods. The reason for such results is using mediuminformative blocks for embedding watermark and adaptive threshold for modification of DHT coefficients. 
Table 1. Comparison of PSNR for the different watermarking algorithms

\begin{tabular}{cccccc}
\hline $\begin{array}{c}\text { Test } \\
\text { image }\end{array}$ & $\begin{array}{c}\text { Results } \\
\text { of } \\
\text { proposed } \\
\text { work }\end{array}$ & $\begin{array}{c}\text { Results } \\
\text { of }\end{array}$ & $\begin{array}{c}\text { Results } \\
\text { of }\end{array}$ & $\begin{array}{c}\text { Results } \\
\text { of }\end{array}$ & $\begin{array}{c}\text { Results } \\
\text { of }\end{array}$ \\
& {$[\mathbf{2 3}]$} & {$[\mathbf{1 3}]$} & {$[\mathbf{2 5}]$} \\
\hline Newyork & 50.08 & 39.78 & 35.55 & 36.76 & 34.87 \\
Boat & 50.26 & 39.62 & 35.76 & 36.12 & 34.56 \\
Opera & 43.89 & 39.56 & 36.24 & 38.24 & 35.45 \\
Bear & 45.78 & 38.49 & 37.89 & 36.24 & 35.61 \\
Pill & 43.18 & 40.12 & 37.21 & 37.21 & 35.76 \\
Lena & 44.82 & 40.02 & 37.45 & 37.85 & 35.67 \\
\hline
\end{tabular}
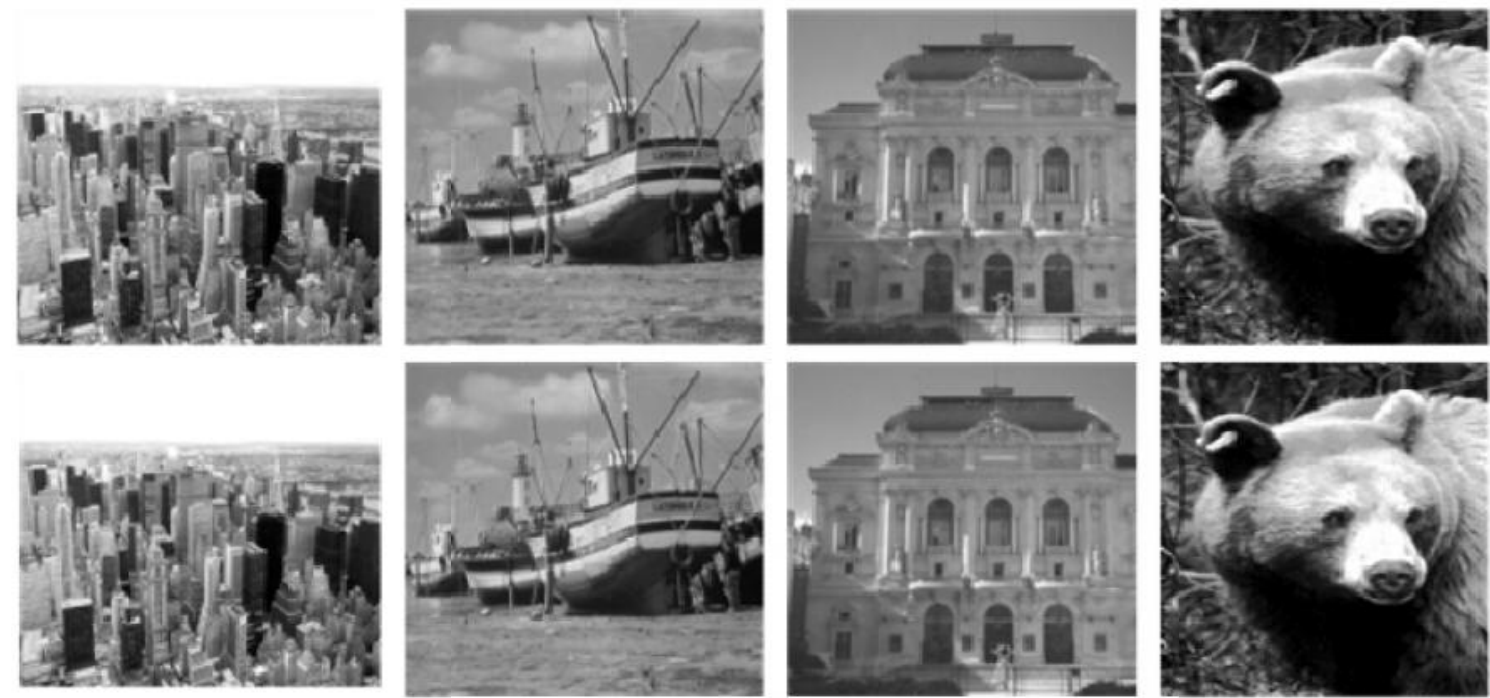

Fig.2.Original images (the first row) and the watermarked images (the second row)

\subsection{Robustness of Proposed Scheme}


To evaluate the robustness of the proposed scheme, each watermarked image was distorted by different types of attacks. After employing the attacks, each watermark was extracted and compared with the original watermark in terms of bit error rate (BER). BER is an objective measure of robustness for watermark image. The ratio of how many bits are detected in error $\left(\mathrm{N}_{\mathrm{ERR}}\right)$ over the total number of bits $\left(\mathrm{N}_{\mathrm{bits}}\right)$ is the BER and it is calculated using Eq.(16).

$$
B E R=\frac{N \frac{E R R}{N} \text { bits }}{B \text { to }}
$$

Number of bits detect in error $\left(\mathrm{N}_{\mathrm{ERR}}\right)$ computed using Eq.(14) wherew $w_{i}^{\prime}$ and $\mathrm{w}_{i}$ are the watermark bits belong to the extracted watermark sequence and in the original watermark sequence respectively.

$$
N_{E R R}=\sum_{=1}^{\prime} \mid-\quad \text { । }
$$

We tested robustness of the proposed scheme under median and Gaussian filters, AWGN, salt \& pepper noise, scaling, rotation, cropping and JPEG and JPEG2000 compression attacks. We applied all these attacks on different test images and BER was computed. BER results for 3 images were shown in Table 2. Robustness of proposed scheme against different attacks is clear from the results.

Figure. 3 show the watermarked Lena images obtained after median filtering using window sizes $(3 \times 3),(5 \times 5),(9 \times 9)$ and $(11 \times 11)$ for Lena, Boat and Opera images, while Figure.4. (a), (b) and (c) show the watermark image after $5^{\circ}, 10^{\circ}$ and $15^{\circ}$ rotation.

Table 2. BER results of proposed scheme under different attacks

\begin{tabular}{lllll}
\hline & $\begin{array}{l}\text { window } \\
\text { size }\end{array}$ & Lena & boat & Opera \\
& $3 \times 3$ & 0.079 & 0.195 & 0.116 \\
Median & $5 \times 5$ & 0.470 & 0.375 & 0.375 \\
Filter & $9 \times 9$ & 0.486 & 0.391 & 0.386 \\
& $9 \times 9$ & & & \\
\hline
\end{tabular}




\begin{tabular}{|c|c|c|c|c|}
\hline \multirow{3}{*}{$\begin{array}{l}\text { Gaussian } \\
\text { filter }\end{array}$} & $3 \times 3$ & 0.128 & 0.102 & 0.115 \\
\hline & $5 \times 5$ & 0.153 & 0.116 & 0.121 \\
\hline & $7 \times 7$ & 0.169 & 0.127 & 0.153 \\
\hline \multirow{3}{*}{ AWGN } & $\sigma n=10$ & 0.110 & $\begin{array}{l}0 . \\
101\end{array}$ & 0.095 \\
\hline & $\sigma n=20$ & 0.208 & 0.185 & 0.198 \\
\hline & $\sigma n=30$ & 0.265 & 0.204 & 0.258 \\
\hline \multirow{5}{*}{$\begin{array}{l}\text { Salt } \\
\text { Pepper }\end{array}$} & $\mathrm{d}=0.01$ & 0.037 & 0.037 & 0.026 \\
\hline & $\mathrm{d}=0.02$ & 0.058 & 0.042 & 0.052 \\
\hline & $\mathrm{d}=0.03$ & 0.084 & 0.095 & 0.116 \\
\hline & $\mathrm{d}=0.04$ & 0.105 & 0.132 & 0.148 \\
\hline & $\mathrm{d}=0.05$ & 0.116 & 0.137 & 0.195 \\
\hline \multirow{5}{*}{$\begin{array}{l}\text { Scaling } \\
\text { factor }\end{array}$} & 1.1 & 0.00 & 0.00 & 0.00 \\
\hline & 1.2 & 0.00 & 0.00 & 0.00 \\
\hline & 1.3 & 0.00 & 0.00 & 0.00 \\
\hline & 1.4 & 0.00 & 0.00 & 0.00 \\
\hline & 1.5 & 0.00 & 0.00 & 0.00 \\
\hline \multirow{5}{*}{$\begin{array}{l}\text { Rotation } \\
\text { angle }\end{array}$} & $1^{\circ}$ & 0.015 & 0.010 & 0.037 \\
\hline & $2^{\circ}$ & 0.021 & 0.010 & 0.058 \\
\hline & $5^{\circ}$ & 0.021 & 0.026 & 0.058 \\
\hline & $10^{\circ}$ & 0.036 & 0.052 & 0.084 \\
\hline & $15^{\circ}$ & 0.047 & 0.058 & 0.108 \\
\hline \multirow{2}{*}{$\begin{array}{l}\text { Cropping } \\
\text { attack }\end{array}$} & $25 \%$ & 0.027 & 0.053 & 0.106 \\
\hline & $50 \%$ & 0.259 & 0.291 & 0.314 \\
\hline \multirow{4}{*}{$\begin{array}{l}\text { JPEG } \\
\text { compression }\end{array}$} & $\mathrm{QF}=90 \%$ & 0 & 0 & 0 \\
\hline & $\mathrm{QF}=70 \%$ & 0 & 0 & 0 \\
\hline & $\mathrm{QF}=50 \%$ & 0.113 & 0.047 & 0.010 \\
\hline & $\mathrm{QF}=30 \%$ & 0.401 & 0.328 & 0.365 \\
\hline \multirow{2}{*}{$\begin{array}{l}\text { JPEG2000 } \\
\text { compression }\end{array}$} & $\mathrm{QF}=90 \%$ & 0 & 0 & 0 \\
\hline & $\mathrm{QF}=70 \%$ & 0.300 & 0.261 & 0.288 \\
\hline
\end{tabular}




$$
\begin{array}{llll}
\mathrm{QF}=50 \% & 0.461 & 0.450 & 0.449 \\
\mathrm{QF}=30 \% & 0.524 & 0.496 & 0.518
\end{array}
$$

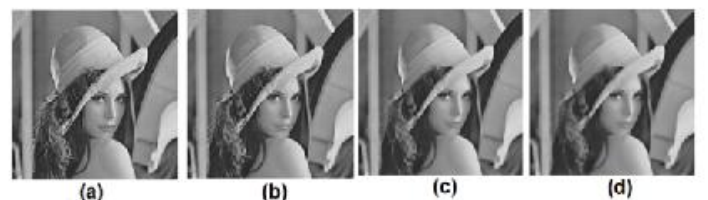

Fig.3. Watermarked image after median filtering with window size: $(a)(3 \times 3)$, (b) $(5$ $\times 5),(c)(9 \times 9)$ and $(d)(11 \times 11)$.

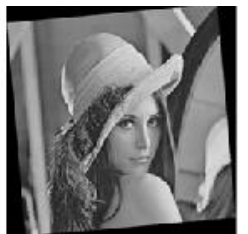

(a)

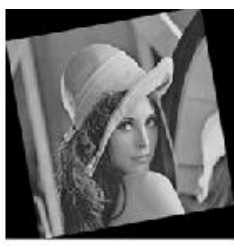

(b)

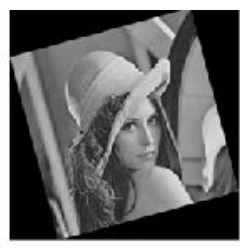

(c)

Fig.4. Watermarked image after (a) $5^{\circ}$ rotation, (b) $10^{\circ}$ rotation and (c) $15^{\circ}$ rotation

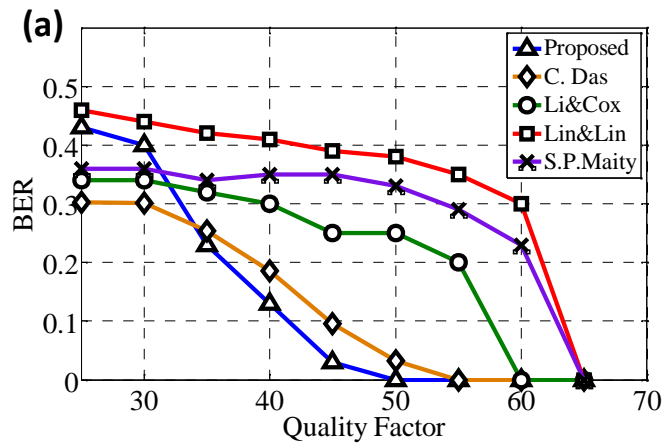




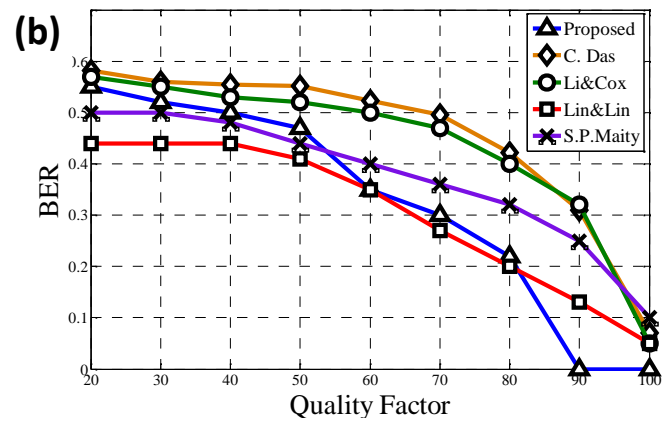

Fig.5. BER performance against lossy (a) JPEG compression operation and (b) JPEG 2000 compression operation

\subsection{Comparison with Other method}

Figure. 5(a) and (b) shows graphically the robustness performance against JPEG and JPEG 2000 compression operations, with comparison with the methods in $[13,17]$. and C.Das[18] methods were based on DCT, Lin and Lin [13] method was a wavelet-based scheme and S.P.Maity[17] was based on DHT. Numerical results showed that the methods in [17-19] offered better robustness for JPEG compared to [13] methods at low quality factor. This was expected as [17, 19] were DHT and DCT based methods, respectively. But the present method showed very good robustness performance at low quality factor. On the other hand, the methods in [13] offered better performance at low quality JPEG 2000 compression compared to [17-19] methods as it was based on wavelet. The proposed method showed comparable performance at low quality factor JPEG2000. Simulation results supports that the selection of Hadamard transform as signal decomposition tool showed better performance with higher payload at low quality compression compared to DCT and DWT, when both JPEG and JPEG 2000 compression operations were taken into consideration.

The robustness of the proposed watermarking scheme was evaluated against several attacks including rotation, scaling, cropping, filtering, JPEG compression and cropping. Table 3 shows BER values of the watermarked images under the aforementioned distortions along with comparison with other methods [13, 17-19]. Minimum values in each row were showen by bold 
numbers. The performance improvement of the proposed method was due to the combined effect of embedding in medium-informative blocks, selecting adaptive thereshold and DHT. One benefit of DHT was binary modulation, which was similar to dither modulation system. Further, DHT offered a form of diversity as coefficients were relatively independent which increased robustness. The aforementioned simulations and analyses have all confirmed that the proposed scheme has high robustness against common geometric transformations, filtering, and compression.

Table 3. Experimental results for the proposed, C.Das[18], Lin and Lin[13] and S.P.Maity[17]

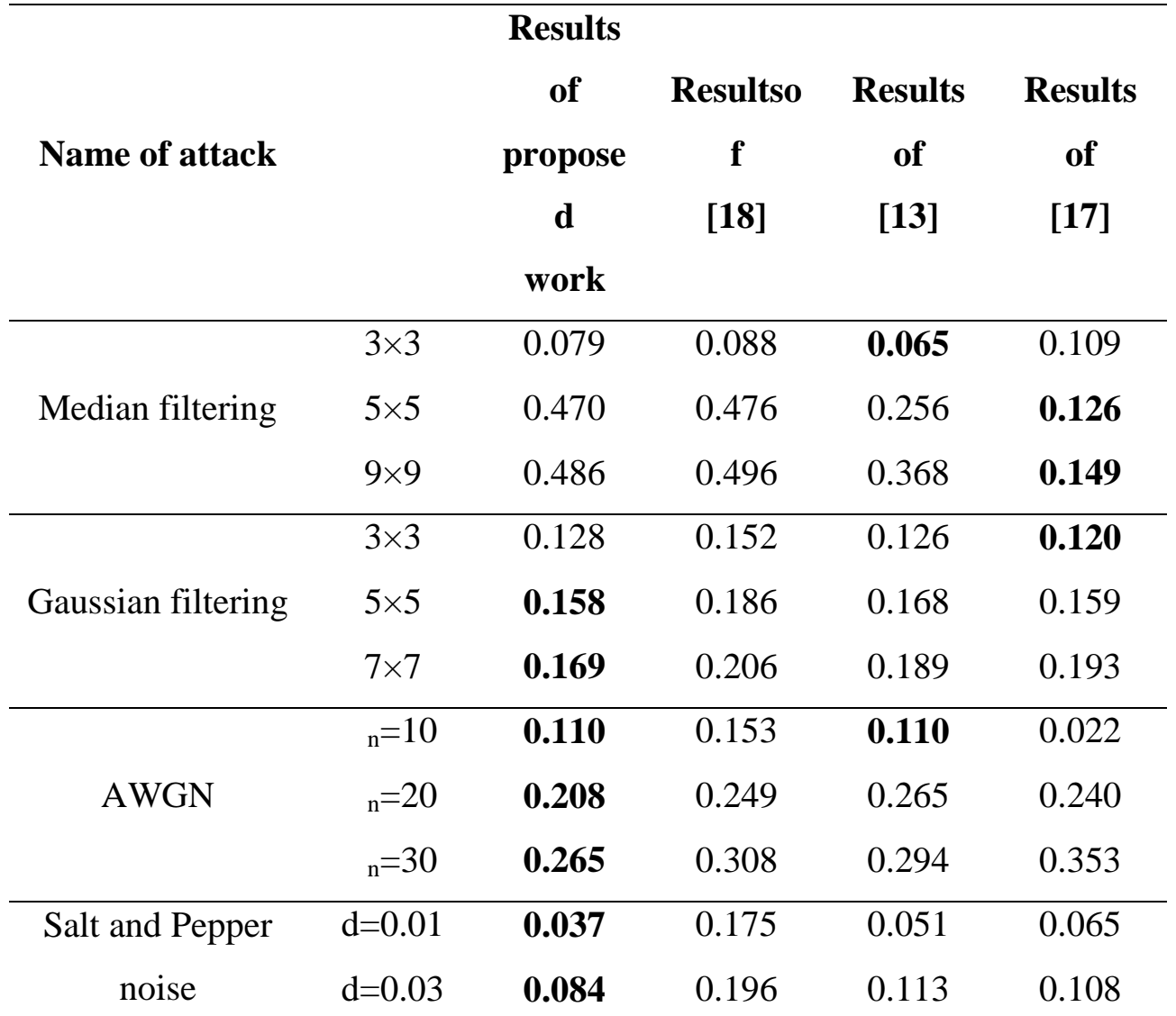




\begin{tabular}{cccccc}
\hline & $\mathrm{d}=0.05$ & $\mathbf{0 . 1 1 6}$ & 0.223 & 0.183 & 0.169 \\
\hline \multirow{3}{*}{ Scaling attack } & 1.1 & $\mathbf{0}$ & $\mathbf{0}$ & 0.023 & 0.020 \\
& 1.3 & $\mathbf{0}$ & 0.023 & 0.083 & 0.102 \\
\multirow{3}{*}{ Rotation } & 1.5 & $\mathbf{0}$ & 0.086 & 0.120 & 0.117 \\
\hline \multirow{3}{*}{ Cropping } & $10^{\circ}$ & $\mathbf{0 . 0 2 1}$ & 0.095 & 0.153 & 0.101 \\
& $15^{\circ}$ & $\mathbf{0 . 0 3 6}$ & 0.123 & 0.213 & 0.188 \\
& $25 \%$ & $\mathbf{0 . 0 2 7}$ & 0.035 & 0.145 & 0.086 \\
& $50 \%$ & $\mathbf{0 . 2 5 9}$ & 0.263 & 0.423 & 0.275 \\
JPEG & $\mathrm{QF}=90 \%$ & 0.456 & 0.489 & 0.563 & $\mathbf{0 . 3 0 6}$ \\
\hline \multirow{2}{*}{ compression } & $\mathrm{QF}=70 \%$ & $\mathbf{0}$ & $\mathbf{0}$ & 0.030 & 0.230 \\
& $\mathrm{QF}=50 \%$ & $\mathbf{0 . 1 1 3}$ & 0.186 & 0.414 & 0.348 \\
\hline \multirow{2}{*}{ JPEG 2000 } & $\mathrm{QF}=90 \%$ & $\mathbf{0}$ & 0.310 & 0.132 & 0.255 \\
& $\mathrm{QF}=70 \%$ & $\mathbf{0 . 3 0 0}$ & 0.495 & 0.321 & 0.361 \\
compression & $\mathrm{QF}=50 \%$ & 0.461 & 0.551 & $\mathbf{0 . 4 1 7}$ & 0.430 \\
\hline
\end{tabular}

\section{CONCLUSIONS}

This paper proposed a perceptually adaptive block-based digital image watermarking algorithm scheme with minimum distortion of the cover image in Hadamard domain. The use of entropy model and selecting adaptive threshold values for each block made a good compromise between robustness and quality of the watermarked image. The algorithm was simple and exploited the inter-block DHT coefficient correlation to embed the watermark by modifying the $4 \times 4$ DHT coefficients of blocks. The use of fast Hadamard transformation reduced the computation cost due to its simplicity of kernel; it improved the robustness against platform independent lossy compression. The direct advantage of this proposed algorithm lies in the improvement of both imperceptibility and robustness. A good quality of watermark has been extracted, with satisfactory BER, from the watermarked image even after performing different image 
manipulation operations like JPEG and JPEG 2000 compression, cropping, rotation, sharpening, etc.

\section{REFRENCES}

[1]E. Muharemagic and B. Furht, "Survey of Watermarking Techniques and Applications," in Multimedia Watermarking Techniques and Applications, D. Kirovski, Ed., ed Boca Raton, Florida: Auerbach Publications, (2006). 91-130.

[2]A. Makur and S. Sethu Selvi" ,Variable dimension vector quantization based image watermarking," Signal Processing,(2001), Vol.81, No.4, 889-893.

[3]E. E. Abdallah, A. B. Hamza, and P. Bhattacharya, "A robust block-based image watermarking scheme using fast Hadamard transform and singular value decomposition," in, 18th International Conference on Pattern Recognition, 2006. ICPR 2006, Hong Kong, 673676, (2006)

[4]J. O'Ruanaidh and T. Pun, "Rotation, translation and scale invariant digital image watermarking," in, International Conference on Image Processing, , 536-536, (1997)

[5]J. J. Ruanaidh and T. Pun, "Rotation, scale and translation invariant spread spectrum digital image watermarking," Signal Processing,(1998), Vol.66, No.3, 303-317.

[6]A. A. N. A. A. A. Ramkumar.M, "A Robust Data Hiding Scheme forImages using DFT," in, International Conference on ImageProcessing, 211-215, (1999)

[7]S. P. Maity and S. Maity, "Multistage Spread Spectrum Watermark Detection Technique Using Fuzzy Logic," IEEE SIGNAL PROCESSING LETTERS,(2009 ,(Vol.16, No.4, 245248.

[8]M. N. Do and M. Vetterli, "The contourlet transform: an efficient directional multiresolution image representation," Image Processing, IEEE Transactions on,(2005), Vol.14, No.12, 2091-2106.

[9]E.Ehsaeyan, "A Robust Image Denoising Technique in the Contourlet Transform Domain," International Journal of Engineering TRANSACTIONS B: Applications,(2015), Vol.28, No.11, 1589-1596.

[10]J. C. Patra, J. E. Phua, and C. Bornand, "A novel DCT domain CRT-based watermarking scheme for image authentication surviving JPEG compression," Digital Signal Processing,(2010), Vol.20, No.6, 1597-1611. 
[11]S. Rawat and B. Raman, "A publicly verifiable lossless watermarking scheme for copyright protection and ownership assertion," AEU-INTERNATIONAL JOURNAL OF ELECTRONICS AND COMMUNICATIONS,(2012), Vol.66, No.11, 955-962.

[12]L. Li, X. Yuan, Z. Lu, and J.-S. Pan, "Rotation invariant watermark embedding based on scale-adapted characteristic regions," Information Sciences,(2010), Vol.180, No.15, 287-5 .2888

[13]T.-C. Lin and C.-M. Lin, "Wavelet-based copyright-protection scheme for digital images based on local features," Information Sciences,(2009), Vol.179, No.19, 3349-3358.

[14]C. Fei, D. Kundur, and R. H. Kwong, "Analysis and design of watermarking algorithms for improved resistance to compression," IEEE TRANSACTIONS ON IMAGE PROCESSING,(2004), Vol.13, No.2, 126-144.

[15]G. Kasana, K. Singhb, and S. S. Bhatia, "Singular Value Decomposition based Steganography Technique for JPEG2000 CompressedImages," International Journal of EngineeringTRANSACTIONS C: Aspects,(2015), Vol.28, No.12,

[16]M. Ramkumar and A. N. Akansu, "Capacity estimates for data hiding in compressed images," IEEE TRANSACTIONS ON IMAGE PROCESSING,(2001), Vol.10, No.8, 1252126.3

[17]S. P. Maity and M. K. Kundu, "DHT domain digital watermarking with low loss in image informations," AEU-INTERNATIONAL JOURNAL OF ELECTRONICS AND COMMUNICATIONS,(2010), Vol.64, No.3, 243-257.

[18]C. Das, S. Panigrahi, V. K. Sharma, and K. K. Mahapatra, "A novel blind robust image watermarking in DCT domain using inter-block coefficient correlation," $\boldsymbol{A E}$ INTERNATIONAL JOURNAL OF ELECTRONICS AND COMMUNICATIONS,(2013), Vol.No.

[19]L. Qiao and I. J. Cox, "Using Perceptual Models to Improve Fidelity and Provide Resistance to Valumetric Scaling for Quantization Index Modulation Watermarking," IEEE TRANSACTIONS ON INFORMATION FORENSICS AND SECURITY,(2007), Vol.No.127-139.

[20]P. Kumsawat, K. Attakitmongcol, and A. Srikaew, "A new approach for optimization in image watermarking by using genetic algorithms," Transaction Signal. Processing.,(2005), Vol.53, No.12, 4707-4719. 
[21]C. E. Shannon, " A mathematical theory of communication," Bell system technical journal,(1948), Vol.27, No.379-423,, 623-656. .

[22]N. R. Pal and S. K. Pal, "Object-background segmentation using new definitions of entropy," Computers and Digital Techniques IEE Proceedings E,(1989), Vol.136, No.4, 284-295.

[23]A. T. Ho, J. Shen, S. H. Tan, and A. C. Kot, "Digital image-inimage watermarking for copyright protection of satellite images using the fast Hadamard transform," IEEE GeoScience and Remote Sensing Symposium,(2002), Vol.6, No.3311-3313.

[24]R. C. Gonzalez and R. E. Woods, Digital Image Processing. New York: Addison-Wesley, .(1992)

[25]R. K. W. I. P. A.H. Paquet, "Wavelet packets-based digital watermarking for image verification and authentication," Signal Processing,(2003), Vol.83, No.2117-2132.

How to cite this article:

Moeinaddini E. A novel perceptually adaptive image watermarking scheme by selecting adaptive threshold in dht domain. J. Fundam. Appl. Sci., 2016, 8(3S), 916-934. 